\title{
Orientation Dependence of the Micro-Pillar Compression Strength in an Electron Beam Melted Ti-6AI-4V Alloy
}

\author{
Jinsen Tian ${ }^{1} \cdot$ Jiang Ma ${ }^{1} \cdot$ Ming $\mathrm{Yan}^{2} \cdot$ Zhuo Chen $^{3} \cdot$ Jun Shen $^{1} \cdot$ Jing $\mathrm{Wu}^{4}$
}

Received: 28 May 2020 / Revised: 22 August 2020 / Accepted: 31 August 2020 / Published online: 11 November 2020

(c) The Chinese Society for Metals (CSM) and Springer-Verlag GmbH Germany, part of Springer Nature 2020

\begin{abstract}
An $\alpha / \beta$ two-phase Ti-6Al-4V alloy was fabricated by electron beam melting to obtain a basketweave structure. The orientation dependence of the mechanical properties of Ti-6Al-4V alloy was studied by micro-pillar compression and post-mortem transmission electron microscopy analysis. The results indicate that different grains have different mechanical responses, and the possible attributions were discussed. Besides the orientation effect, due to the limited volumes of micropillars, the size of the $\alpha$ phases, dispersion of the $\beta$ phases, and the presence of the free dislocation path also affect the mechanical properties of the micropillars to a large extent. Although no direct link was discovered between the mechanical properties and the parent $\beta$ orientations, this work provided a promising method to further study the anisotropic mechanical behavior in Ti-6Al-4V alloy.
\end{abstract}

Keywords Electron beam melting $(\mathrm{EBM}) \cdot \mathrm{Ti}-6 \mathrm{Al}-4 \mathrm{~V} \cdot$ Micro-pillar compression $\cdot$ Orientation dependence $\cdot$ Mechanical properties

\section{Introduction}

Ti-6Al-4V (Ti64) alloy is widely used in aerospace industries and biomedical fields due to its light weight, high specific strength and good bio-compatibility [1]. However, achieving the complex geometries of aerospace components or the required efficiency for customized bio-implants for humans is difficult using conventional subtractive methods. Additive manufacturing (AM) technique, which allows

Available online at http://link.springer.com/journal/40195.

Jun Shen

junshen@szu.edu.cn

$\triangle$ Jing Wu

wujinguob@163.com

1 College of Mechatronics and Control Engineering, Shenzhen University, Shenzhen 518060, China

2 Department of Materials Science and Engineering and Shenzhen Key Laboratory for Additive Manufacturing of High-Performance Materials, Southern University of Science and Technology, Shenzhen 518055, China

3 Aerospace Hiwing (Harbin) Titanium Industrial Co., Ltd., Harbin 150028, China

4 Cryo-EM Center, Southern University of Science and Technology, Shenzhen 518055, China layer-by-layer building of complex functional parts designed in a 3D model, shows great promise in the construction of aerospace components and medical devices [2-4]. Electron beam melting (EBM) is one of the powder bed AM techniques that utilize electron beam as heating source and has been extensively studied for Ti64 alloy [5-14].

Mechanical properties comparable with those of the aswrought alloys can be achieved through EBM process for Ti64 alloy by careful selection of processing parameters, such as scanning strategy and input energy density $[11,15$, 16]. However, controlling the microstructural consistency throughout a whole component is difficult. Hrabe and Quinn [17] reported that the horizontal built part of EBM-built Ti64 alloy has a significantly larger elongation than the vertical built part, but yield strength is the same in both parts. Zhao et al. [18] reported higher strength and better ductility in the vertically built part compared with the horizontally built part of the EBM Ti64 alloy. Although inconsistent conclusions have been drawn in different studies, the mechanical performance of EBM-processed Ti64 alloy varies with the part/building orientation.

In addition to existing defects (pores), elongated parent $\beta$ grains and microstructural texture are responsible for the anisotropic mechanical behavior in AM-fabricated alloy [13, $19,20]$. Most of parent $\beta$ grains exhibit a coarse columnar structure and a dominant $\langle 001\rangle$ growth direction $[12,14$, 
21]. However, the direct link between the texture/orientation variation and mechanical properties remains unclear. The influence of part locations and defects should be excluded to establish the correlation between the parent $\beta$ orientation and mechanical properties. Single crystals can be traditionally grown to study the orientation effect; however, the process is very time consuming. At present, microsized single crystals, which can be machined using focus ion beam (FIB) and tested typically under the electron microscopes, are commonly used to study the mechanical responses of various materials. In the present work, micro-pillar compression will be employed to investigate the orientation-dependent strength of EBM-fabricated Ti64 alloy within an individual parent $\beta$ grain.

\section{Experimental}

Ti64 powders with size of $45-105 \mu \mathrm{m}$ were supplied by AP\&C, Canada. The chemical compositions of the powders are listed in Table 1. The cylindrical samples were built using Acram EBM Q20. The horizontal section of the as-fabricated sample was mechanically cut and polished for microstructural analysis using a Tescan Mira-3 scanning electron microscope
(SEM). The thin foil of the as-fabricated sample for transmission electron microscopy (TEM) was prepared using twin-jet electropolishing (Struers tenupol-5) in a solution containing $5 \% \mathrm{HClO}_{4}, 35 \%$ butanol and $60 \%$ methanol and observed using ThermoFisher Talos F200.

Prior to micro-pillar milling, electron backscattered diffraction (EBSD) analysis was carried out in an FEI G4 Xe plasma FIB equipped with Bruker e-flash detector to identify the orientation of parent $\beta$ grains. Since residual $\beta$ phase is very thin and difficult to detect during large area scan, only $\alpha$ variants were selected for indexing. The normal directions of the $\beta$ grains were recovered from the EBSD results. The micro-pillars with a typical size of $\Phi 18 \mu \mathrm{m} \times 40 \mu \mathrm{m}$ were machined using plasma FIB with high tension of $30 \mathrm{kV}$ and current of $15 \mathrm{nA}$ finishing. The resulting pillars are tapered cylinders where the top diameter is smaller than the bottom diameter. The tapering angle is around $2.8^{\circ}$. The micropillars were compressed using Alemnis compression system installed inside the plasma FIB, and a displacement control mode was employed with a loading strain rate of $1 \times 10^{-3} \mathrm{~s}^{-1}$ and a $20 \%$ strain. The compressed pillars were then imaged for slip trace. TEM thin foils were lifted out and thinned from compressed pillars using FEI Helios 600i FIB and FEI Helios G4 FIB and then characterized using FEI Tecnai F30.

Table 1 Chemical composition of the Ti64 powders (wt.\%)

\begin{tabular}{llllllllll}
\hline $\mathrm{C}$ & $\mathrm{O}$ & $\mathrm{N}$ & $\mathrm{H}$ & $\mathrm{Fe}$ & $\mathrm{Al}$ & $\mathrm{V}$ & $\mathrm{Y}$ & $\mathrm{Others}$ & $\mathrm{Ti}$ \\
\hline 0.01 & 0.09 & 0.02 & 0.003 & 0.18 & 6.42 & 4.13 & 0.001 & $<0.4$ & Balance \\
\hline
\end{tabular}
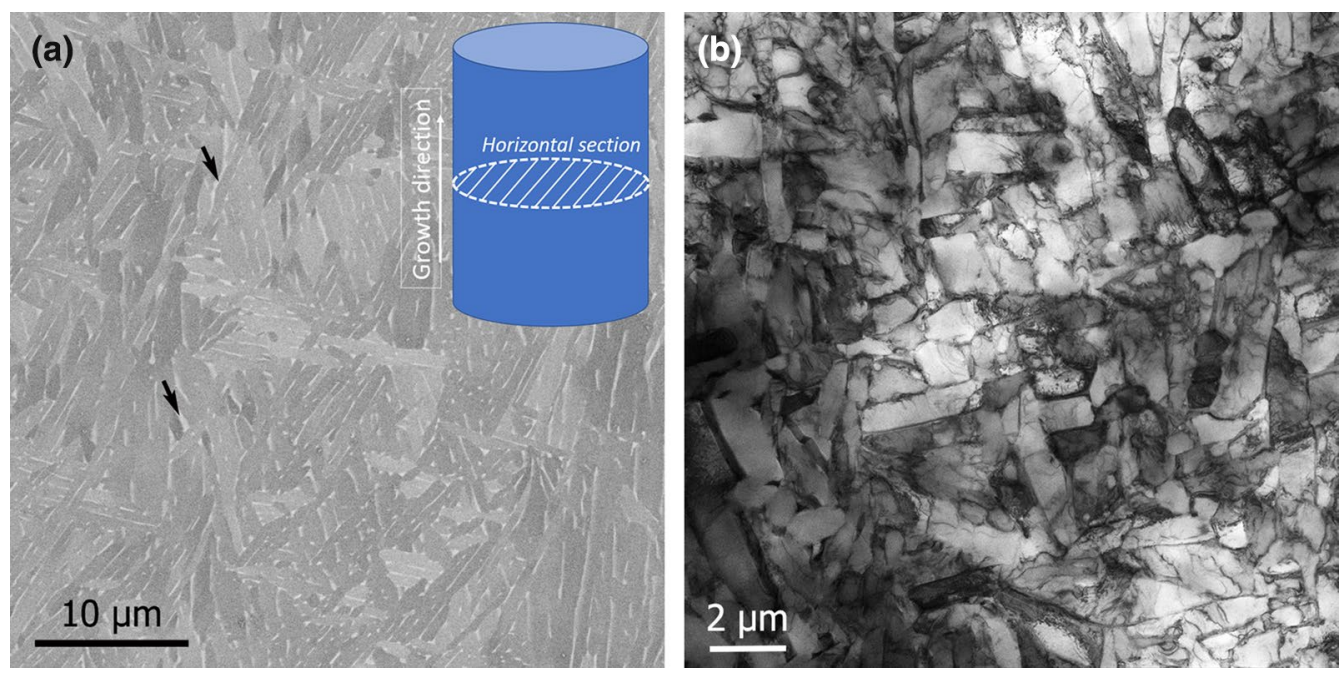

Fig. 1 a BSE image of the horizontal section showing the structure of EBM-fabricated Ti64 alloy; inset showing the schematic drawing of the sample section; b bright-field TEM image showing the overall microstructure and dislocations 


\section{Results}

Figure 1 shows a typical horizontal section of the as-fabricated Ti64 sample. The backscattered electron (BSE) image in Fig. 1a shows a basketweave structure, where fine $\alpha$ plates precipitate from the high-temperature $\beta$ phase. The residual $\beta$ phase possesses thin white layers as indicated by arrows. Some $\alpha$ plates have brightness that differs from the other plates, indicating orientation difference among different variants. Small groups of $\alpha$ plates with similar orientation are identified. Figure $1 \mathrm{~b}$ shows the bright-field TEM image of the horizontal section. A high density of dislocations is observed inside the $\alpha$ grains and at the interface of $\alpha / \beta$ plates. The basketweave morphology and high-density dislocations are caused by the high cooling rate during the EBM process.

A large horizontal section of the as-fabricated sample was characterized by EBSD to show the parent $\beta$ grain in Fig. 2. The orientation map shows the $\alpha$ variants individually oriented in different directions, but over a large area, they share the same orientation, which indicates that they were transformed from the same high-temperature $\beta$ grain. Distinguishing the parent $\beta$ grain boundaries without reconstruction is difficult; however, reasonable assumption can be made based on the orientation of the $\alpha$ variants. For example, seven regions are labeled in Fig. 2 a to represent different $\beta$ grains, and their corresponding $\{0001\}$ pole figures of $\alpha$ variants are shown in Fig. $2 b$. The parent $\beta$ grains have considerably larger grain sizes, typically few hundreds of microns, than the transformed $\alpha$ variants. The

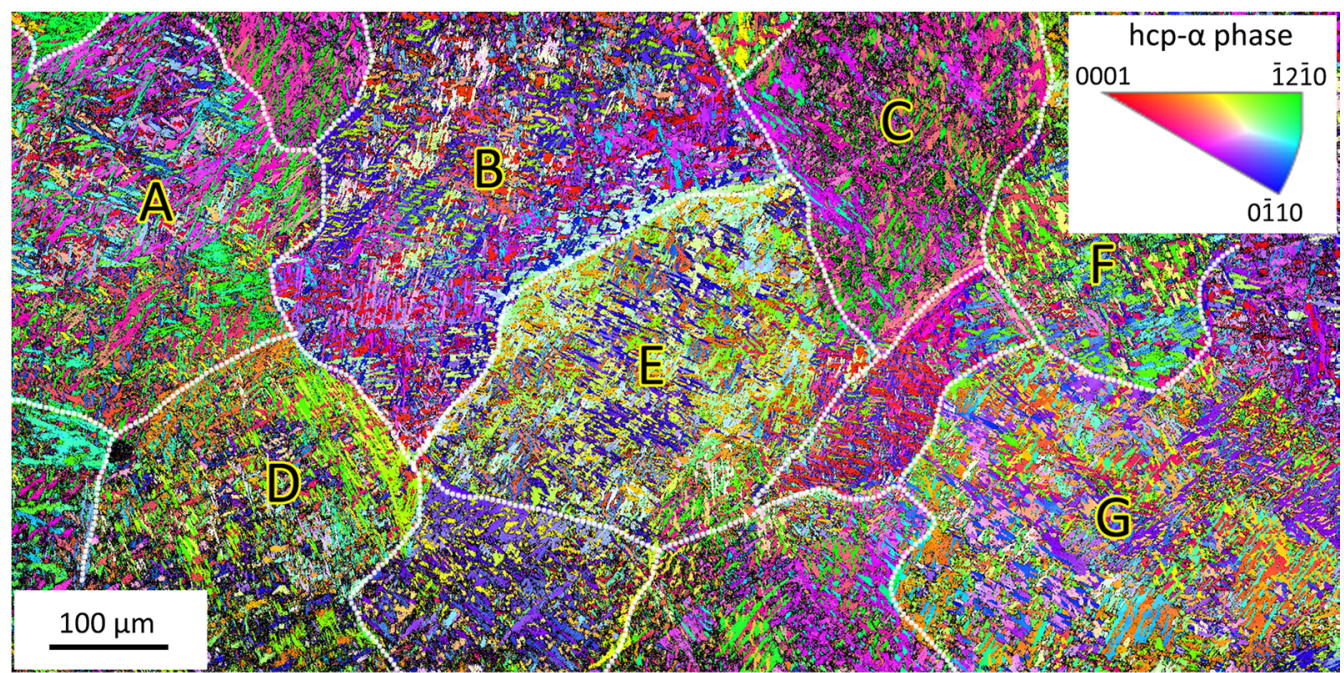

(a)

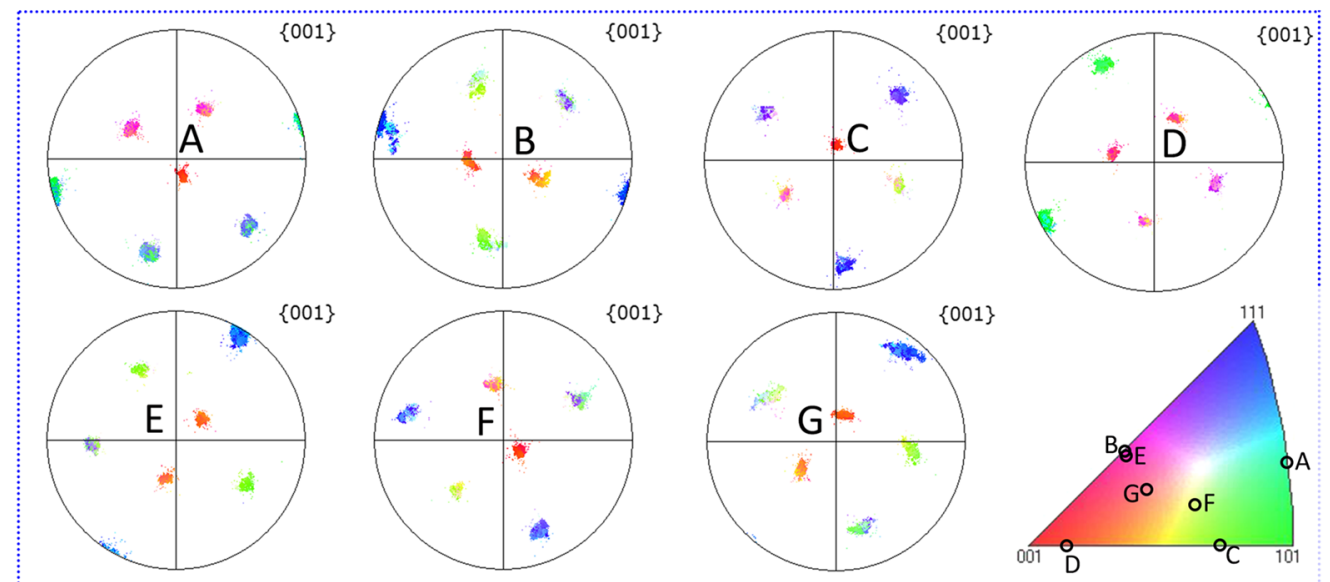

(b)

Fig. 2 a EBSD orientation map of the horizontal section of the as-fabricated sample; only $\alpha$ phase is used for phase indexation. The parent $\beta$ boundaries were indicated by white dashed line. b Pole figure of [0001] zone axis corresponding to the area labeled in a. The inverse pole figure indicates the normal directions of the transformed $\beta$ grains, which is represented by small circles 
pole figures indicate that at least six variants exist in a single $\beta$ grain. The normal direction of the parent $\beta$ grain is calculated based on Burgers relationship between $\alpha / \beta$ phases, and they are shown as small circles in the inverse pole figure (Fig. 2b). A dominant $\langle 001\rangle$ growth direction of the parent $\beta$ grains was reported in literature $[12,14$, 21]; however, in the current study, a specific texture cannot be identified due to the limited sampling.

Micro-pillars were machined from these seven regions with different $\beta$ loading directions, and the compression curves are shown in Fig. 3 along with a typical image of the micro-pillar before compression. It is clear that different compression strengths can be obtained from different parent $\beta$ grains, but it does not necessarily depend on the parent $\beta$ orientation, i.e. the grain $\mathrm{B}$ and grain $\mathrm{E}$ share the same normal direction, but the yield strength has a $10 \%$ difference. On the other hand, micro-pillars having different normal directions of parent $\beta$ grains can have similar compression strength, e.g. grain B, F and G. The corresponding $0.2 \%$ yield strengths are listed in Table 2 along with $2 \%$ and $10 \%$ flow stresses. The highest yield strength was observed

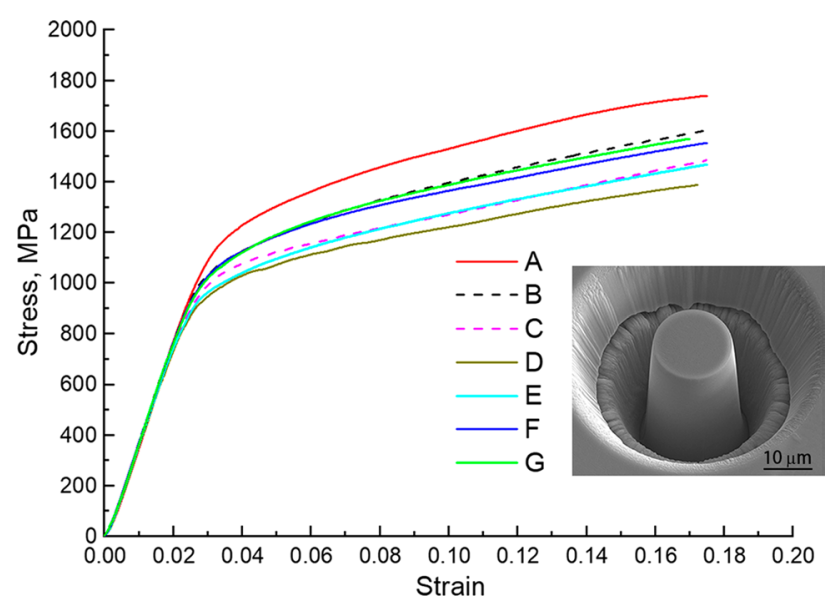

Fig. 3 Stress-strain curves of the compressed micro-pillars corresponding to parent $\beta$ grains in Fig. 2 in the pillar from orientation $\mathrm{A}$, which is $25 \%$ higher than the lowest yield strength in the pillar with orientation D. The overall yield strength is within 0.86-1.07 GPa. When dealing with the microsized samples, the size effect is always considered. Liu et al. [22] reported that the size effect in $\mathrm{Mg}$ can be neglected when the pillar size is larger than $10 \mu \mathrm{m}$. Nonetheless, the tensile tests for the bulk sample reported by Hrabe and Quinn [9] show comparable yield strength values ranging from $0.93-0.95 \mathrm{GPa}$. This suggests that the mechanical properties of the current micro-pillars are representative of the bulk samples.

The micro-pillars after compression were characterized by SEM, and the results are shown in Fig. 4. After compressed with a $20 \%$ strain, all the pillars have multiple slip steps on the sample surface. The largest shear step was observed in pillar D which has the lowest yield strength. Compared with pillar D, the slip steps in the pillar A are relatively small and interrupted by other slip systems. There is no obvious difference in the compressed morphology between pillar B and pillar E despite they have a slight difference in yield strength.

So, TEM analysis is necessary for the deformed structures. Figure 5a shows a TEM lamella lifted out from pillar $\mathrm{D}$, and the viewing direction is indicated with respect to the pillar. Figure $5 \mathrm{~b}$ shows a narrow shear band in the middle with severe deformation in contrast to the neighboring materials which appear more homogeneously deformed. This shear band corresponds to the large step in pillar D shown in Fig. 4. Assume that the shear band is edge on, and the angle between slip band and loading direction is $50^{\circ}$. Using STEM diffraction, it is able to examine the orientation across the shear band, and it turns out the materials inside the shear band belong to the same $\alpha$ variant with slight deviation caused by the deformation. The orientation of the $\alpha$ variant in the shear band (indicated by the circle in Fig. 5c) was determined by diffraction pattern (Fig. 5d) to be hcp structure and [0001] orientation. This suggests that the shear band is close the $(1 \overline{1} 00)$ prismatic plane.
Table 2 Compression strengths of the micro-pillars calculated from Fig. 4

\begin{tabular}{lllll}
\hline Micro-pillars & $\begin{array}{l}0.2 \% \text { yield } \\
\text { strength, GPa }\end{array}$ & $\begin{array}{l}2 \% \text { flow stress, } \\
\text { GPa }\end{array}$ & $\begin{array}{l}10 \% \text { flow stress, } \\
\text { GPa }\end{array}$ & $\begin{array}{l}\text { Minimum strength needed } \\
\text { for dislocation activation* }\end{array}$ \\
\hline $\mathrm{A}$ & 1.07 & 1.32 & 1.67 & 0.83 \\
$\mathrm{~B}$ & 0.97 & 1.19 & 1.51 & 0.84 \\
$\mathrm{C}$ & 0.92 & 1.12 & 1.37 & 0.81 \\
$\mathrm{D}$ & 0.86 & 1.06 & 1.30 & 0.92 \\
$\mathrm{E}$ & 0.88 & 1.08 & 1.37 & 0.92 \\
$\mathrm{~F}$ & 0.97 & 1.18 & 1.46 & 0.91 \\
$\mathrm{G}$ & 0.95 & 1.19 & 1.49 & 0.81 \\
\hline
\end{tabular}

*This value is calculated based on the loading direction and Schmid factor of each $\alpha$ variant, and it indicates the lowest stress needed for activating at least one prismatic $\langle a\rangle$ slip system in a single variant 

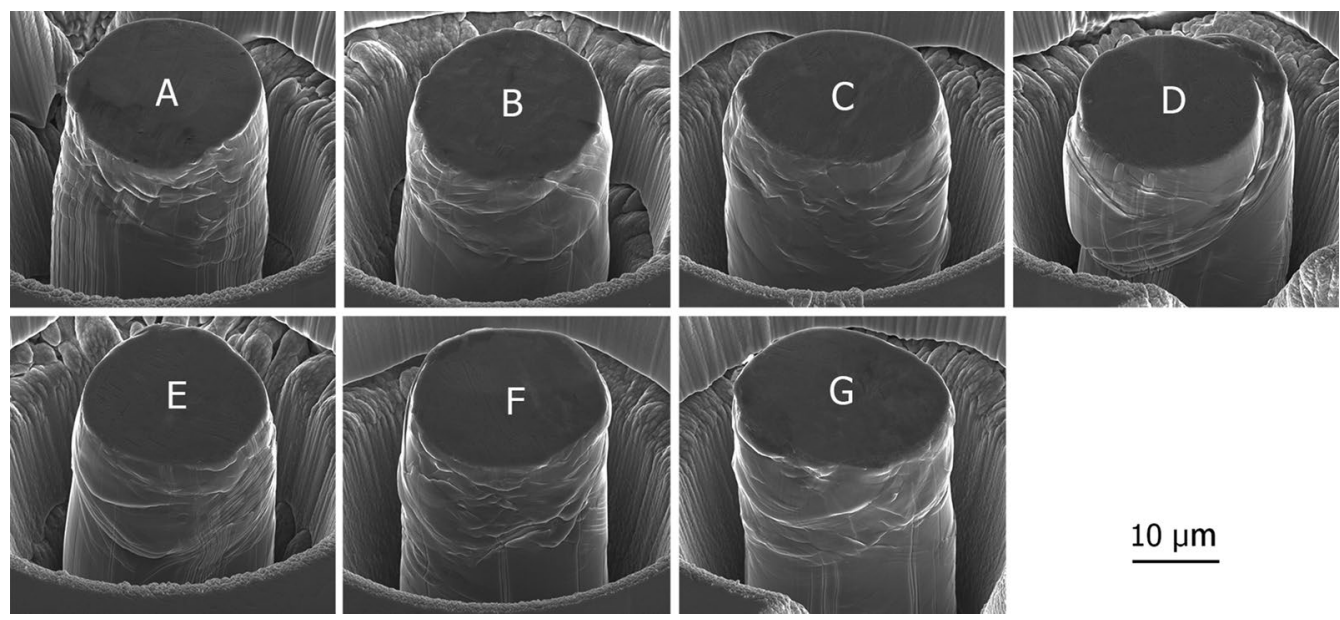

$10 \mu \mathrm{m}$

Fig. 4 SEM images of the compressed pillars taken at stage tilt angle of $40^{\circ}$. The letters represent the pillars from corresponding grains
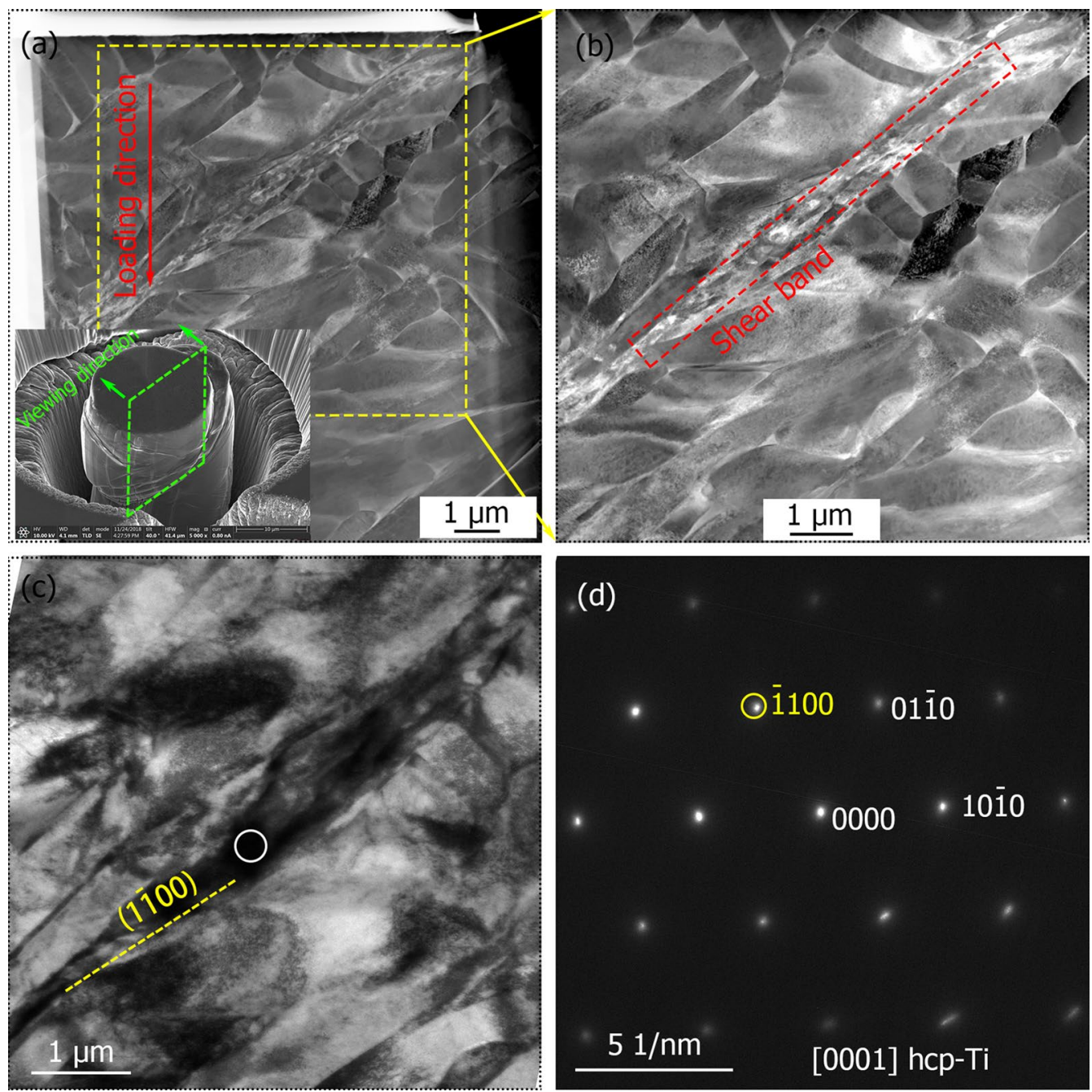

Fig. 5 TEM results from compressed pillar D: a STEM HAADF image showing the overview deformed structure; b a magnified STEM image showing a localized shear band; c, d TEM bright-field image and the corresponding selected area diffraction patterns of the shear band region 

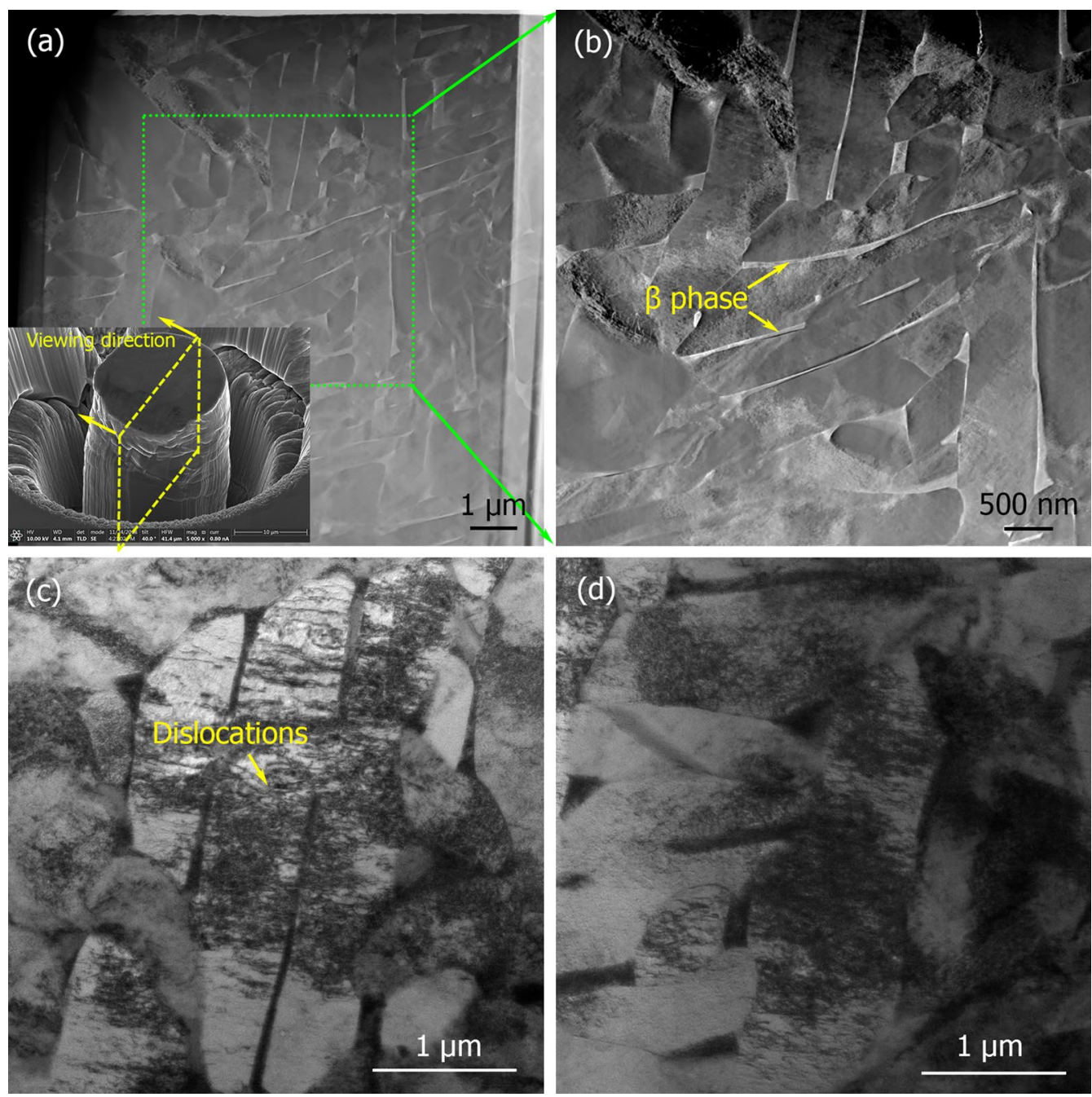

Fig. 6 Deformed morphologies from compressed pillar A: a STEM overview image showing the deformed structure, the inset showing the SEM image and the relative view direction for TEM observation; $\mathbf{b}$ magnified STEM image; $\mathbf{c}, \mathbf{d}$ TEM bright-field images showing the dense dislocations in the $\alpha$ grains

The deformed structure of pillar A is shown in Fig. 6. There is no obvious shear band, indicating a homogeneous deformation. Thicker $\beta$ phase was observed compared with grain D. The TEM images show high density of dislocations everywhere. Figure $6 \mathrm{c}$ shows a large colony which includes multiple $\alpha$ variants with the same orientation but separated by $\beta$ phase. The dislocations show similar morphology in this colony under the current beam condition.

Figure 7 compared the deformed structure from pillar $\mathrm{E}$ and pillar B, which share the similar orientation but with different yield strengths. In Grain E, there are very large $\alpha$ variants (the yellow outlined region in Fig. 7), and no $\beta$ phase was observed inside these variants. High density of dislocations appear as white lines present in these $\alpha$ variants and multiple slip systems are activated (indicated by red arrows). In comparison, pillar B shows smaller $\alpha$ variant size and dispersed $\beta$ phase (indicated by blue rectangular box). 

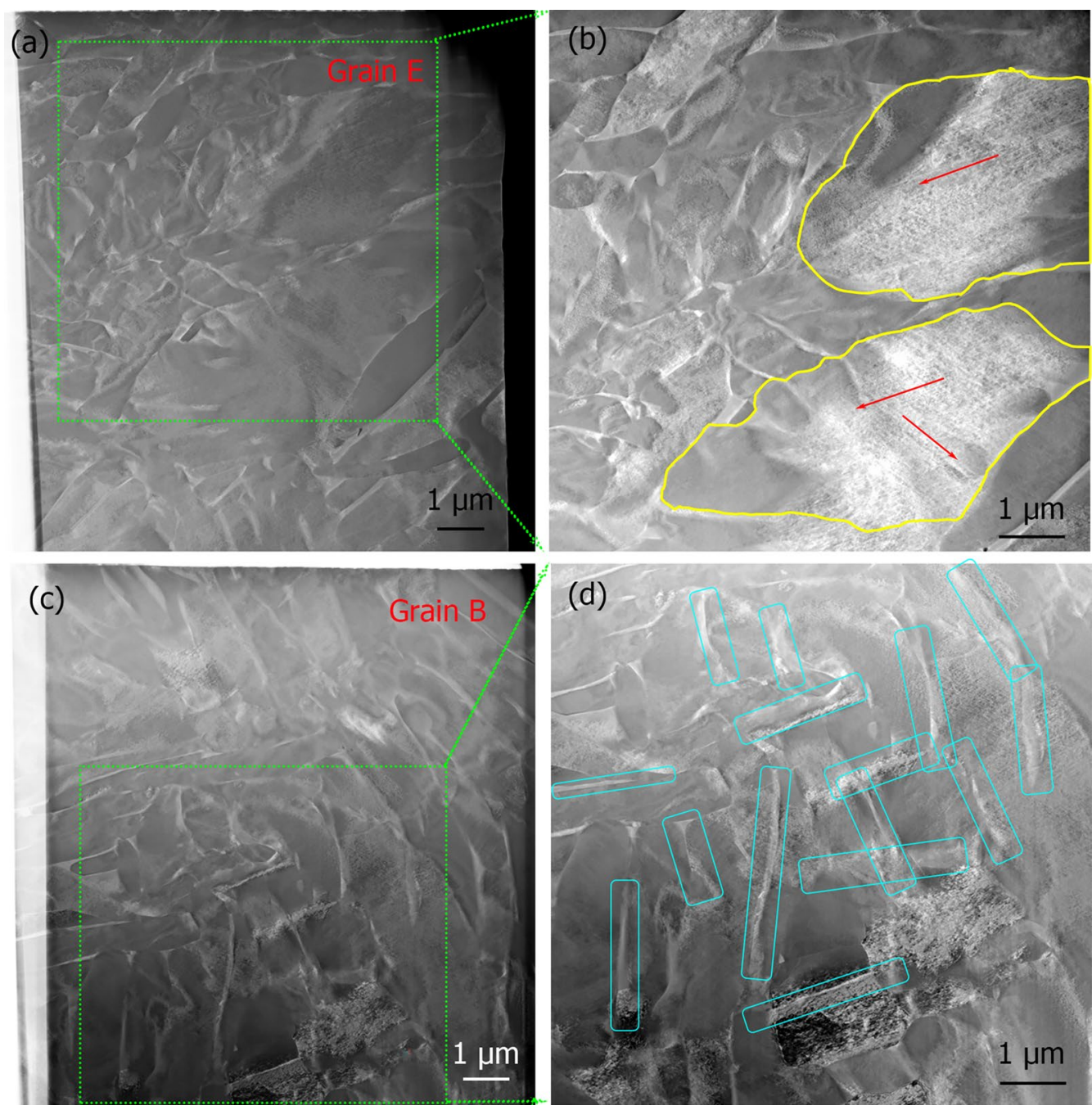

Fig. 7 a, b STEM high angle annular dark field (HAADF) images from compressed pillar E showing large $\alpha$ variants, the dense dislocations appear as white lines and red arrows indicate the slip directions of few slip systems inside two large $\alpha$ variants; $\mathbf{c}, \mathbf{d}$ STEM HAADF images from compressed pillar B revealing more dispersed $\beta$ phase (indicated by blue boxes)

\section{Discussion}

\subsection{The role of orientation on the strength of micropillars}

To understand the underlying mechanism of the mechanical behaviors, the orientation dependence will first be considered. It is well known that the yield strength of single crystals is mainly determined by two key factors: i.e. CRSS and Schmid factor. The minimum strength required for yielding in a single crystal phase can be calculated using $\sigma_{\mathrm{y}}=\sigma_{\mathrm{CRSS}} / \mathrm{m}$. It is more complicated in the current Ti64 alloy where both $\alpha$ and $\beta$ phases present. $\alpha$ phase is the major component transferred from parent $\beta$, and its deformation behavior has been widely studied. Generally, the pyramidal $\langle c+a\rangle$ type slip system has a considerably higher CRSS compared with $\langle a\rangle$ type. Jones and
Hutchinson [23] reported that the most easily activated slip system of $\alpha$ phase at room temperature and under compression is prismatic $\langle a\rangle$ slip (CRSS of approximately $392 \mathrm{MPa}$ ), followed by pyramidal $\langle a\rangle$ slip (CRSS of approximately $404 \mathrm{MPa}$ ) and basal $\langle a\rangle$ slip (CRSS of approximately $444 \mathrm{MPa}$ ).

If only $\alpha$ variants are considered, the minimum stress required to initiate $\langle a\rangle$ slip systems for each micro-pillar is estimated in Table 2 based on the CRSS and Schmid factor of each variant. Overall, the measured yield strength range $(0.86-1.07 \mathrm{GPa})$ is higher than the estimated value range $(0.81-0.92 \mathrm{GPa})$. But there are exceptions, i.e. the estimated minimum strengths to activate $\langle a\rangle$ slip systems in grain $\mathrm{D}$ and $\mathrm{E}$ are higher than the measured yield strengths. It appears that no clear connection exists between the experimental results and the estimated yield strengths for $\alpha$ variants. This also indicates that no obvious linkage is found 
between the mechanical response of the Ti64 and the orientation of the parent $\beta$ phase. This may be due to the polycrystalline nature of $\alpha$ phase, the presence of the residual $\beta$ grains and the interfaces.

\subsection{Interface effect on the strength of micropillars}

Two important interfaces, i.e., $\alpha / \alpha$ and $\alpha / \beta$ boundaries, play significant roles in strengthening the Ti64 alloys. Similar with grain boundaries, these boundaries act as obstacles hindering the dislocation movement. However, unlike grain boundaries, certain orientation relationship exists in $\alpha / \alpha$ and $\alpha / \beta$ interface. For $\alpha / \alpha$ boundaries, six types are obtained in the current basketweave structure due to the high cooling rate. For example, Wang et al. [24] defined an $\alpha / \alpha$ interface sharing a type $2\left([11 \overline{2} 0] / 60^{\circ}\right)$ boundary with a $[11 \overline{2} 0]$ common axis parallel to $[111]_{\beta}$ zone axis of the parent $\beta$ phase, and the misorientation angle is $60^{\circ} .\langle a\rangle$ type dislocation can easily cross slip through this type of boundary when the loading direction is appropriate. However, for other types, piling up of dislocations at the interface boundary is expected. For $\alpha / \beta$ boundaries, due to the Burgers relationship, only one type of $\langle a\rangle$ dislocations $\left(a_{1}=1 / 3[2 \overline{110}]\right)$ can transmit through the interface boundary given the shallow angle between the broad face and slip plane [25]. Dislocations with Burgers vector of $a_{2}=1 / 3[1210]$ or $a_{3}=1 / 3[11 \overline{2} 0]$ will glide a large angle at the interface and do not easily transmit through. It eventually results in dislocation pile-ups at the interface boundary. Typical interactions between dislocations and the $\beta$ phase are observed.
Figure 8a shows that at least two groups of dislocation lines were present in $\alpha$ phase under the current beam condition. Group 1 dislocations sheared through the $\beta$ phase during the deformation, and $\beta$ phase becomes discontinuous (Fig. 8b). Gaps are also observed between different parts of $\beta$ phase and may be caused by movements of group 2 dislocations.

In bulk samples, Galarraga et al. [26] reported that the hardness of the Ti64 decreases with increasing $\alpha$ variant size. Similar trend is also observed in the current study, e.g. pillar $\mathrm{E}$ has larger $\alpha$ variant size and less $\beta$ phase comparing to the pillar B (Figs. 6 and 7), and this may result in lower strength. But different from bulk samples, the micro-pillar has limited volume where dislocation may move freely without interacting with interfaces before leaving the sample surface. Although in average the thickness of the $\alpha$ variant is small, its length and width are comparable with the micropillar size, indicating that free dislocation path may exist. Pillar D is such a case, and the thin variant is cross the whole pillar and has $1 / 3[11 \overline{2} 0](1 \overline{1} 00)$ slip system with Schmid factor around 0.45 . The slip plane is parallel to the variant which indicates that once the slip is activated, the dislocations glide on prismatic slip can move freely without encounter with $\alpha / \alpha$ and $\alpha / \beta$ boundaries until slip out of the sample surface. And this free dislocation path is likely responsible for the lowest yield strength observed in pillar $D$. So in micro-sized pillars, the local difference in variant sizes, the volume fraction of the $\beta$ phase and also dislocation free path are all critical in varying the mechanical properties.
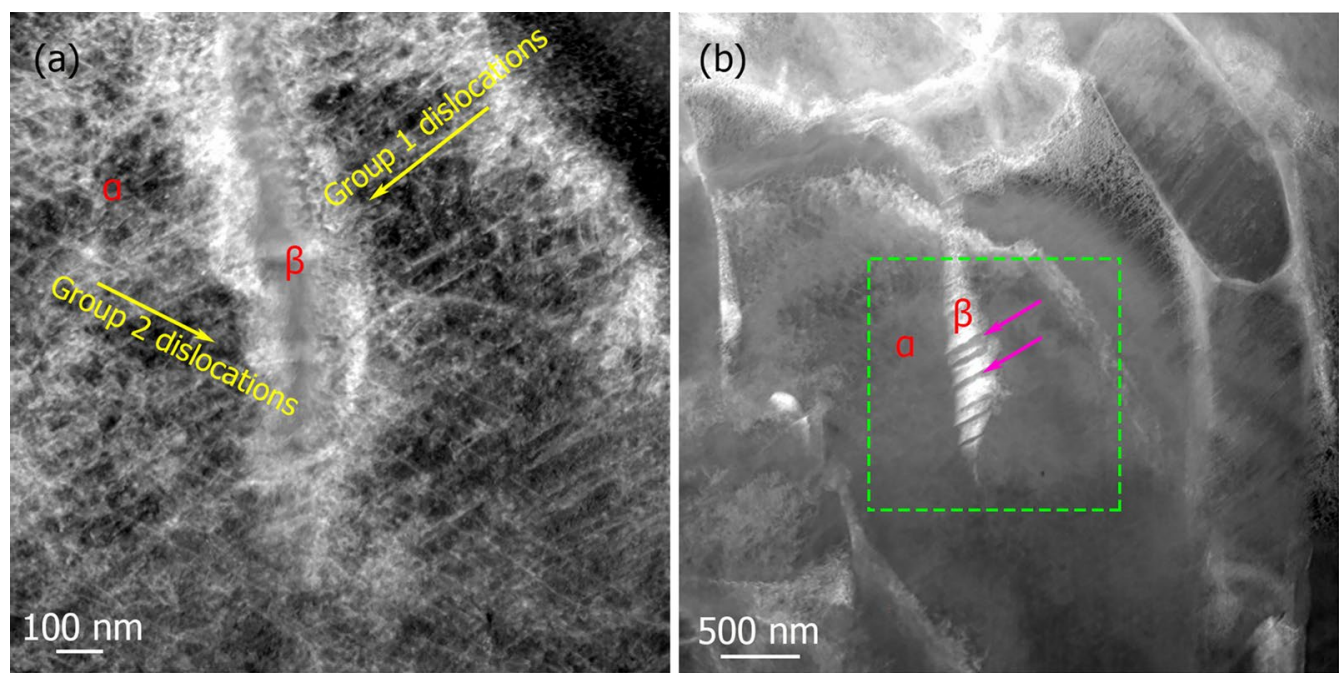

Fig. 8 a STEM image of deformed pillar B showing the interaction between $\beta$ phase and dislocations in $\alpha$ phase. The dislocations appear as white lines and yellow arrows represent the slip directions of two groups of dislocations. The same region under different beam conditions is presented by the green box in $\mathbf{b}$. The $\beta$ phases were segmented by dislocations transmitted through, and the magenta arrows show the gaps between $\beta$ segments caused by deformation 


\section{Conclusion}

The microstructure and orientation-dependent mechanical properties of the as-fabricated Ti64 alloy by EBM were investigated. The as-fabricated alloy contains a basketweave structure with more than six variants present inside a single $\beta$ parent grain due to the high cooling rate. The micro-pillars of $\alpha / \beta$ two-phase alloy from different parent $\beta$ orientations are compressed, and different mechanical responses occurred, but no direct link is obtained between mechanical properties and parent $\beta$ orientation. The TEM results confirm that the mechanical properties of the micro-pillars may be attributed to size of $\alpha$ grains, the dispersion of $\alpha / \beta$ interface boundaries and the free dislocation path due to limited volume of micro-pillars.

Acknowledgement The authors acknowledge the assistance of SUStech Core Research Facilities and SUStech Cryo-EM facility center.

\section{References}

[1] C. Veiga, J.P. Davim, A.J.R. Loureiro, Rev. Adv. Mater. Sci. 32, 133 (2012)

[2] L.L. Xing, C.C. Zhao, H. Chen, Z.J. Shen, W. Liu, Acta Metall. Sin. -Engl. Lett. 33, 981 (2020)

[3] D.D. Gu, W. Meiners, K. Wissenbach, R. Poprawe, Int. Mater. Rev. 57, 133 (2012)

[4] W.E. Frazier, J. Mater. Eng. Perform. 23, 1917 (2014)

[5] G. Del Guercio, M. Galati, A. Saboori, P. Fino, L. Iuliano, Acta Metall. Sin. -Engl. Lett. 33, 183 (2020)

[6] C. Wei, X. Ma, X. Yang, M. Zhou, C. Wang, Y. Zheng, W. Zhang, Z. Li, Mater. Lett. 221, 111 (2018)
[7] Y. Liu, J. Zhang, S.J. Li, W.T. Hou, H. Wang, Q.S. Xu, Y.L. Hao, R. Yang, Acta Metall. Sin. -Engl. Lett. 30, 1163 (2017)

[8] J. Karlsson, A. Snis, H. Engqvist, J. Lausmaa, J. Mater. Process. Technol. 213, 2109 (2013)

[9] N. Hrabe, T. Quinn, Mater. Sci. Eng. A 573, 264 (2013)

[10] T. Sun, Y. Liu, S.J. Li, J.P. Li, Acta Metall. Sin. -Engl. Lett. 32, 869 (2019)

[11] L.E. Murr, E.V. Esquivel, S.A. Quinones, S.M. Gaytan, M.I. Lopez, E.Y. Martinez, F. Medina, D.H. Hernandez, E. Martinez, J.L. Martinez, S.W. Stafford, D.K. Brown, T. Hoppe, W. Meyers, U. Lindhe, R.B. Wicker, Mater. Charact. 60, 96 (2009)

[12] S.S. Al-Bermani, M.L. Blackmore, W. Zhang, I.J.M. Todd, Metall. Mater. Trans. A 41, 3422 (2010)

[13] J. Bruno, A. Rochman, G. Cassar, J. Mater. Eng. Perform. 26, 692 (2017)

[14] A.A. Antonysamy, J. Meyer, P.B. Prangnell, Mater. Charact. 84, $153(2013)$

[15] A.N. Kalinyuk, N.P. Trigub, V.N. Zamkov, O.M. Ivasishin, P.E. Markovsky, R.V. Teliovich, S.L. Semiatin, Mater. Sci. Eng. A 346, $178(2003)$

[16] L.E. Murr, S.A. Quinones, S.M. Gaytan, M.I. Lopez, A. Rodela, E.Y. Martinez, D.H. Hernandez, E. Martinez, F. Medina, R.B. Wicker, J. Mech. Behav. Biomed. Mater. 2, 20 (2009)

[17] N. Hrabe, T. Quinn, Mater. Sci. Eng. A 573, 271 (2013)

[18] X. Zhao, S. Li, M. Zhang, Y. Liu, T.B. Sercombe, S. Wang, Y. Hao, R. Yang, L.E. Murr, Mater. Des. 95, 21 (2016)

[19] M. Simonelli, Y.Y. Tse, C. Tuck, Mater. Sci. Eng. A 616, 1 (2014)

[20] B.E. Carroll, T.A. Palmer, A.M. Beese, Acta Mater. 87, 309 (2015)

[21] C. de Formanoir, S. Michotte, O. Rigo, L. Germain, S. Godet, Mater. Sci. Eng. A 652, 105 (2016)

[22] Y. Liu, N. Li, M. Arul Kumar, S. Pathak, J. Wang, R.J. McCabe, N.A. Mara, C.N. Tomé, Acta Mater. 135, 411 (2017)

[23] I.P. Jones, W.B. Hutchinson, Acta Metall. 29, 951 (1981)

[24] S.C. Wang, M. Aindow, M.J. Starink, Acta Mater. 51, 2485 (2003)

[25] S. Suri, G.B. Viswanathan, T. Neeraj, D.H. Hou, M.J. Mills, Acta Mater. 47, 1019 (1999)

[26] H. Galarraga, R.J. Warren, D.A. Lados, R.R. Dehoff, M.M. Kirka, P. Nandwana, Mater. Sci. Eng. A 685, 417 (2017) 